
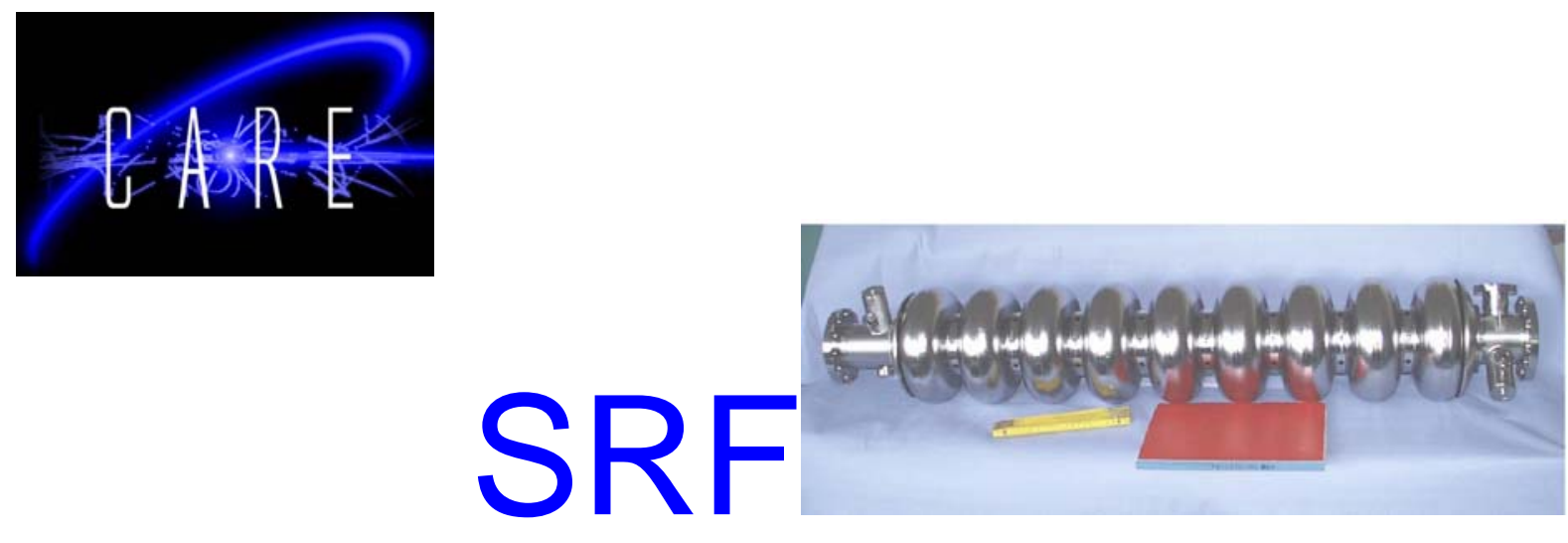

\title{
New method for RF field amplitude and phase calibration in FLASH accelerator
}

\author{
P. Pawlik, M. Grecki,
}

TECHNICAL UNIVERSITY OF LODZ, POLAND

\begin{abstract}
This paper presents new method for RF field amplitude and phase calibration in FLASH accelerator. Theold method for calibration had some disadvantages, which are absent in a new method. The concept is based ondetection and measurement of single bunch induced transients. These transients are very small and it is not possible todetect them without special hardware. The paper presents the new developed system for a single bunch inducedtransient detection with a measurement results that proof that the system is working correctly and can be used forcalibration.
\end{abstract}

Contribution to the MIXDES, Gdynia (Poland)

Work supported by the European Community-Research Infrastructure Activity under the FP6 "Structuring the European Research Area" programme (CARE, contract number RII3-CT2003-506395) 


\title{
NEW METHOD FOR RF FIELD AMPLITUDE AND PHASE CALIBRATION IN FLASH ACCELERATOR
}

\author{
P. PAWLiK ${ }^{1}$, M. GRECKI ${ }^{1}$, S. SimRocK ${ }^{2}$ \\ ${ }^{1}$ TECHNICAL UNIVERSITY OF LODZ, POLAND \\ ${ }^{2}$ DeUTSCHES ELEKTRONEN-SYNCHROTRON, GERMANY
}

\section{KEYWORDS: FLASH, Beam, Transient, Comb Filter, Calibration}

\begin{abstract}
This paper presents new method for RF field amplitude and phase calibration in FLASH accelerator. The old method for calibration had some disadvantages, which are absent in a new method. The concept is based on detection and measurement of single bunch induced transients. These transients are very small and it is not possible to detect them without special hardware. The paper presents the new developed system for a single bunch induced transient detection with a measurement results that proof that the system is working correctly and can be used for calibration.
\end{abstract}

\section{INTRODUCTION}

The FLASH accelerator uses 9-cell superconducting cavities made from Nobium to accelerate beam electrons. Beam is a train of electron bunches with a single bunch charge in a range $0.5 \mathrm{nC}-3 \mathrm{nC}$ [1]. Time space between every bunch is $1 \mu \mathrm{s}$. Bunch accelerating voltage strongly depends on a phase between beam and the $1.3 \mathrm{GHz}$ RF electromagnetic field inside a cavity. It relates in a cosine function. For $0^{\circ}$ beam receives the highest energy and for $180^{\circ}$ beam is maximally decelerated. Which means that it is important to properly adjust RF field phase with respect to the beam phase. This adjustment is called phase calibration.

The RF field is generated by klystrons. Each klystron drives single module, which consists of 8 cavities. A sum vector of fields from all cavities in a single module controls the field generated by klystron. In order for a sum control to work properly it is required to set scaling factors of the RF field ADCs. These factors tell how field measured with ADC in one cavity relates to the field in a different one within a single module. Values for these factors are taken during amplitude calibration. Currently used method for amplitude and phase calibration is based on high charge beam induced transient detection. This method does not require any special equipment but it has some disadvantages. Main disadvantage is that it is required to stop normal accelerator operation and set special conditions for calibration. For this reason it can be done only very rarely. Setting special conditions for calibration reduces measurement accuracy. For different conditions accelerator parameters can be slightly different. Another disadvantage is that this method has a harming influence on electronics inside the tunnel. Accelerator parameters change with temperature and time. Calibration factors after a period of time since last calibration become inaccurate or even incorrect. It is necessary to have a method that could be done online during normal accelerator operation and which could detect any changes in beam phase or in field magnitudes continuously.

Method that solves problems existing in currently used approach is based on a single bunch induced transient detection. The basic concept is to detect and measure single RF field changes induced by single bunch. Main difficulty in this method is that these changes are very small they are around 1000 times smaller than the absolute RF field magnitude and cannot be detected without special hardware. However it has advantages over currently used method. Calibration can be done during normal accelerator operation. It is not necessary to set special conditions hence the measurement can be done constantly and can be more accurate. It does not have any harming influence on electronics inside the tunnel.

\section{DETECTION CONCEPT}

Detection of a small $1.3 \mathrm{GHz}$ RF field changes that are 1000 smaller then the field requires carrier filtering. The method, which attenuates by around $100 \mathrm{~dB}$ carrier while leaving small transients unattenuated, is based on subtraction of two identical signals where one is delayed with respect to the other one. Subtraction is done in a passive microwave circuit as a sum of two vectors where one is shifted in phase by $180^{\circ}$ with respect to the second one. One of the signals is delayed by 20ns and the second has magnitude and phase adjusted with variable attenuator and variable phase shifter to get $180^{\circ}$ phase difference at $1.3 \mathrm{GHz}$ and to have the same magnitudes. Such signals are summed with a power combiner to produce the result of the subtraction.

Signals within such a filter and the output signal are presented in Fig. 1. In a first stage both signals at the input of a combiner actual and delayed one have the value of the RF field. The output signal in this case equals filter adjustment error. In a perfect case this output should be zero but in reality phase cannot be adjusted perfectly to $180^{\circ}$ and magnitudes to be exactly the same hence the output is a filter adjustment error, which is around $100 \mathrm{~dB}$ smaller then the carrier for sufficiently adjusted filter. At the second stage actual signal equals a sum of the RF field in cavity and the transient. Subtraction of actual and delayed signal equals in this case a sum o filter adjustment error and transient. Third stage is when both signals actual and 
delayed one have the same value that is a sum of the RF field and transient signals. In this instance the filter output equals a filter adjustment error. To conclude, the output of a filter contains pulse that has an information about the RF field transient induced by a single bunch in a form of a pulse with a length which is the same as a delay in a filter delay line.

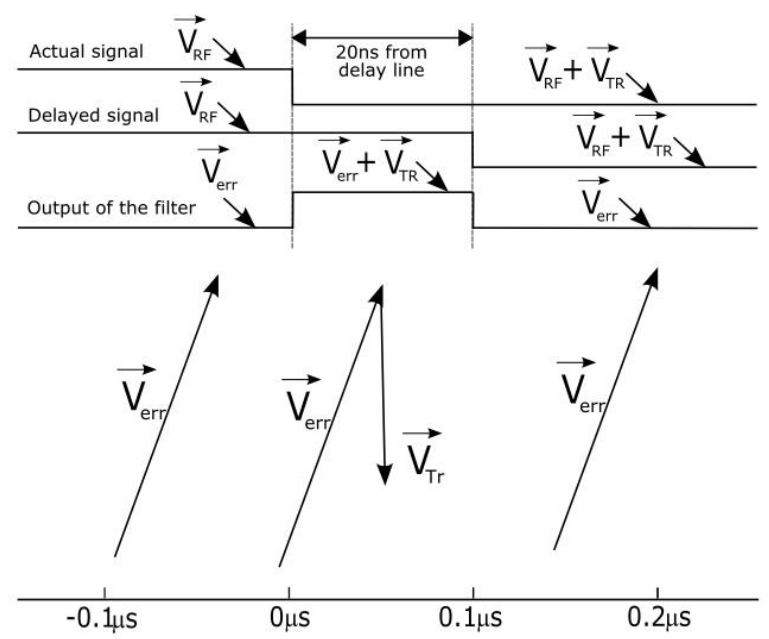

Fig. 1 Single bunch induced transient detection concept

\section{HARDWARE}

The purpose of a single bunch induced transient detection system is to measure a beam phase with a transient magnitude and export them to DOOCS accelerator control system. System that was already developed and is currently working which targets this aim is presented in Fig. 2 and Fig. 3.

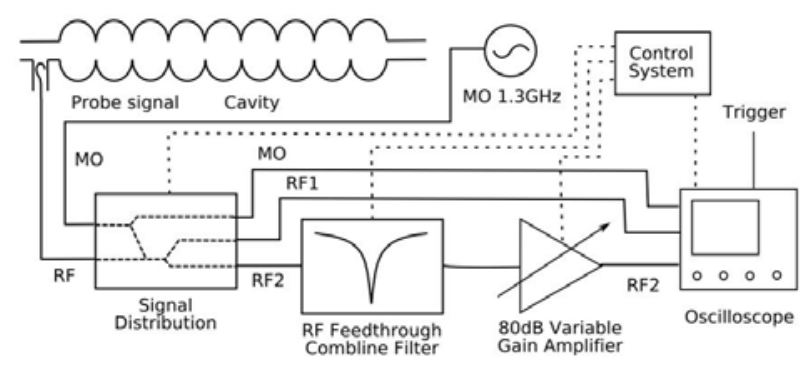

Fig. 2 Single bunch induced transient detection system

It consists of several components: signal distribution board, RF Feedthrough Combline Filter, $80 \mathrm{~dB}$ variable gain amplifier, oscilloscope and a control system. Signal distribution board distributes RF signals to other components. It delivers signals from the cavity probe during normal system operation and $1.3 \mathrm{GHz}$ master oscillator during system calibration. RF Feedthrough Combline Filter filters signal from the cavity probe. It attenuates carrier and leaves transients in a form of unattenuated pulses. Variable gain amplifier amplifies filtered transients by around $80 \mathrm{~dB}$. Oscilloscope digitizes two RF signals, RF1 from the cavity probe and RF2 from the filter. These signals are later used in a Matlab software installed on the oscilloscope to calculate vectors. These vectors are later divided one by the other and multiplied by a calibration factors in order to get the relative magnitude and phase between them. The result is a single bunch induced transient vector calculated with respect to the RF field in the cavity.

Signal distribution board, filter and amplifier are controlled by a control system. This system is composed of ADC, DAC and digital IO modules installed in a VME board. DOOCS server installed on another VME board with a SPARC processor and Solaris operating system controls these modules. A Matlab script running on the oscilloscope through the Internet controls DOOCS server, which controls mentioned modules.

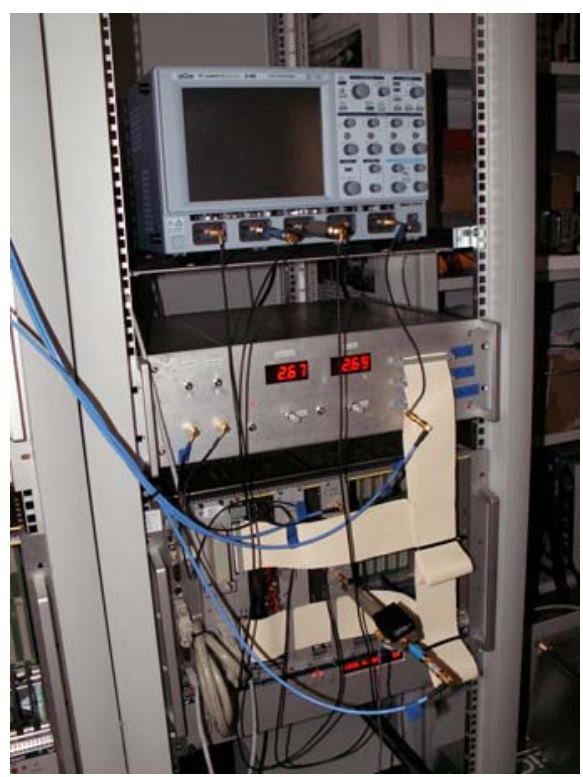

Fig. 3 Single bunch induced transient detection system

The most difficult part in the system is a Feedforwad Combline Filter. It is difficult to adjust its magnitude and phase with an accuracy of $0,001^{\circ}$ in phase and $1 e-5$ in magnitude to get $100 \mathrm{~dB}$ carrier attenuation. Filter ones adjusted becomes detuned when the temperature changes. Temperature changes cause phase shifts and also attenuation changes in a filter, which in turn detunes filter. The solution to this problem is a special circuit for filter adjustment with a part consisting of mechanical phase shifter and attenuator for rough adjustment and additional part with vector modulator for fine-tuning. Where output signal of vector modulator is attenuated by $70 \mathrm{~dB}$ and summed with a signal from mechanical attenuator and phase shifter. In this way magnitude and phase adjustments with accuracy of $0,001^{\circ}$ in phase and 1e-5 in magnitude is easy. Unfortunately vector modulator can only adjust phase and magnitude in a limited range. When the temperature changes are too large filter goes out of working range of the vector modulator. For this reason it is necessary to roughly stabilize filter thermally to keep it in a working range of a vector modulator.

\section{DOOCS INTERFACE}

Measurement results and all necessary data is stored in a DOOCS server. System uses four DOOCS servers: 
adc_server,

ipdigi_server,

dac08_server,

rftransient_server. First three mentioned servers control ADC's, digital IO and DAC modules. Last server rftransient_server stores data related to transient measurement like beam phase, filter adjustment error, etc. DOOCS was chosen as a system for data storing and for transient detection control because this is a control system used for a whole FLASH accelerator.

Control of a Single Bunch Induced Transient Detection System is done with DOOCS interface. Main panel for this interface is presented in Fig. 1. In a picture one can see table with measured beam phase and transient magnitude. This table has values from single bunch measurement and a value calculated as an average over 100 samples. On the right side of this table there is a figure, which presents beam charge. In the centre there are two figure with captured transients, one of them presents real part of data and the second one imaginary. On the bottom part of this panel are buttons for invoking another panels for: variable gain amplifier control, filter control, main calibration and IQ modulator calibration.

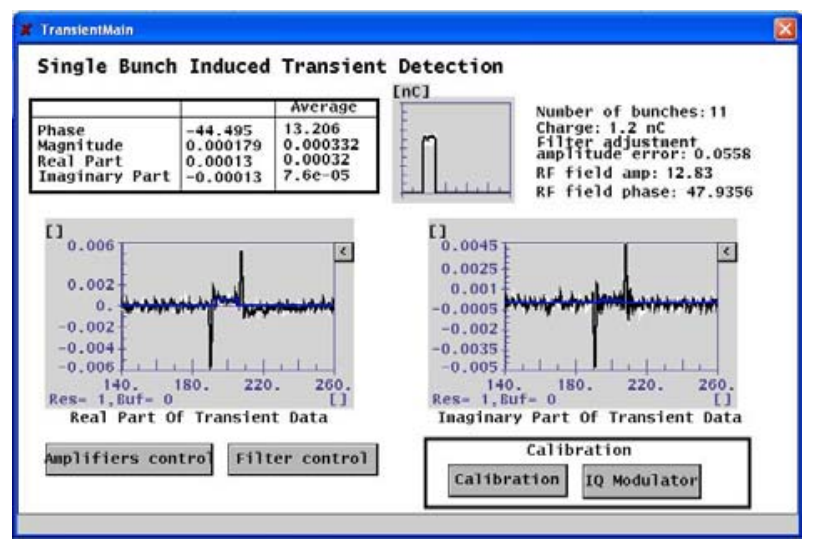

Fig. 4 Main DOOCS panel for transient detection

\section{MEASUREMENT RESULTS}

The application of the presented system is a calibration. To assure that this system is working correctly many tests have been performed during special accelerator study periods. The latest results are presented below. Tests have been performed to check if measurements conform theory.

First test in Fig. 5 compares two different methods for beam phase measurement. One of the methods is an old method used for calibration that uses high charge transient. The second measurement is done with the Single Bunch Induced Transient Detection System. Presented result shows that both measurements conform each other. This proofs that the new method is working correctly.

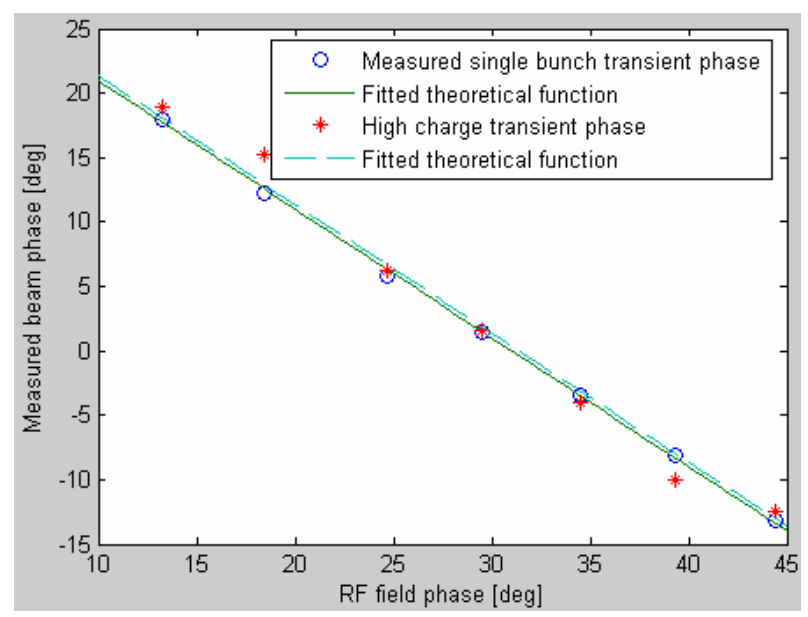

Fig. 5 Beam phase measurement

Second measurement checks whether transient magnitude has value, which is expected by a theory. During this test several measurements have been performed for different beam charges starting from $0.5 \mathrm{nC}$ and ending in $3 \mathrm{nC}$ for the same beam phase. Theoretically equation (1) derived from a cavity model presented in [1] is expected to show the relation between single bunch charge and transient magnitude.

$$
\left|V_{t r}\right|=4 \pi \cdot f_{1 / 2} \cdot R_{L} \cdot q \cdot\left|V_{\text {field }}\right|^{-1}
$$

In Fig. 6 one can see that measurement results are correct with a theoretical equation. Line was fitted to results with parameters that are expected by the theory: $\mathrm{f}_{1 / 2}=208.9 \mathrm{~Hz}, \mathrm{R}_{\mathrm{L}}=1.562 \mathrm{G} \Omega, \mathrm{V}_{\text {field }}=13 \mathrm{MV} / \mathrm{m}$.

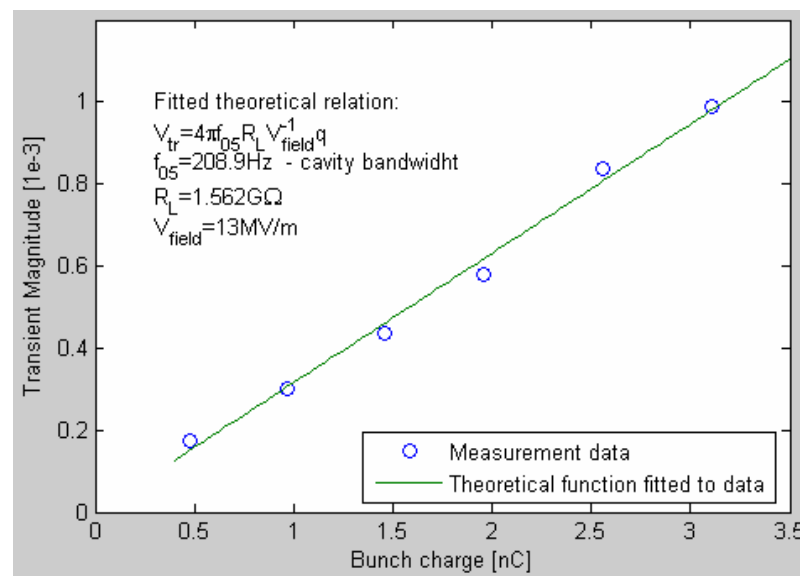

Fig. 6 Transient magnitude measurement

Last measurement checks whether beam energy is changing in a correct relation to the beam phase. During this measurement phase was changed only in one cavity in a module. Beam phase in this cavity was measured with Transient Detection System. For every phase in this cavity energy at the end of the module was measured. In Fig. 7 the $\mathrm{x}$-axis presents measured beam phase and $y$-axis presents measured beam energy at the end of the module. Theoretically the energy should change as a cosine function with maximum at $0^{\circ}$ phase. Energy measured for $90^{\circ}$ should equal the energy received from 7 cavities in a module. As it can be seen 
measurement results conform expectations. Cosine function fitted to results has maximum at around $0^{\circ}$ beam phase and for $90^{\circ}$ it has an energy that is expected to be received from 7 cavities in a module.

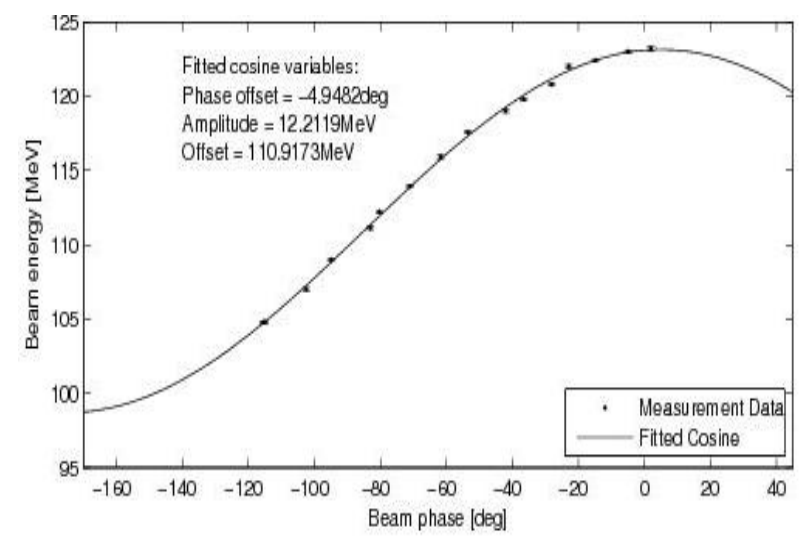

Fig. 7 Beam energy measurement

\section{CONCLUSION}

Presented system for transient detection is currently working in DESY Hamburg. It has advantages over an old method used for calibration. It can be used during normal accelerator operation. It does not require setting special conditions in accelerator. It can be used constantly, which reduces errors that are caused by changing parameters of the accelerator in time. It has no harming influence on electronics inside the accelerator tunnel.

The system has a nice graphical user interface developed in DOOCS interface for controlling it. It is already connected to the interface normally used to control FLASH accelerator. This interface enables transient measurement, system control and calibration. Performed tests during accelerator studies periods showed that the system is working in conformance with theory and it could be used as a new method for the RF electromagnetic field calibration.

\section{THE AUTHORS}

Mgr Pawel Pawlik and Dr Mariusz Grecki are with the Depertment of Microelectronics and Computer Science , Technical University of Lodz, Lodz, Poland

E-mail: pawel.pawlik@dmcs.p.lodz.pl. mgrecki@dmcs.p.lodz.pl

Dr Stefan Simrock is with the Deusches Electronen Synchrotron, Hamburg, Germany

E-mail: stefan.simrock@desy.de

\section{ACKNOWLEDGEMENTS}

We acknowledge the support of the European Community-Research Infrastructure Activity under the FP6 „Structuring the European Research Area” program (CARE, contract number RII3-CT-2003-506395), and Polish National Science Council Grant "138/E370/SPB/6.PR UE/DIE 354/2004-2007”

\section{REFERENCES}

[1] T. Schilcher, "Vector Sum Control of Pulsed Accelerating Fields in Lorentz Force Detuned Superconducting Cavities", PhD thesis

[2] P. Pawlik, M. Grecki, S. Simrock "System for High Resolution Detection of Beam Induced Transients in RF Signals" 12 th International Conference MIXDES 2005, Kraków, Poland, 23-25 June, pp. 815 - 820, vol. 2, ISBN 83-919289-9-3

[3] P. Pawlik, M. Grecki, S. Simrock „Single Bunch Transient Detection for The Beam Phase Measurement in Superconducting Accelerators" Proceedings of DIPAC 2005, Lyon, France, http://accelconf.web.cern.ch/AccelConf/d05/PAPE RS/POM031.PDF

[4] S.N. Simrock, T. Schilcher DESY “Transient Beam Loading Based Calibration Of the Vector-Sum For The Tesla Test Facility”, DESY http://accelconf.web.cern.ch/AccelConf/e96/PAPE RS/THPL/THP025L.PDF

[5] P. Pawlik, S. Simrock, H.C. Weddig "Simulation And Measurement Of An RF Notch Filter For An Electron Beam Single Bunch Detector", MIXDES 2004, Szczecin 24-26 June 2004 Poland, pp. 430434 